$\angle$ Research Square

\title{
The application of WT1 and P53/Ki-67 to predict the recurrence of epithelial ovarian cancer
}

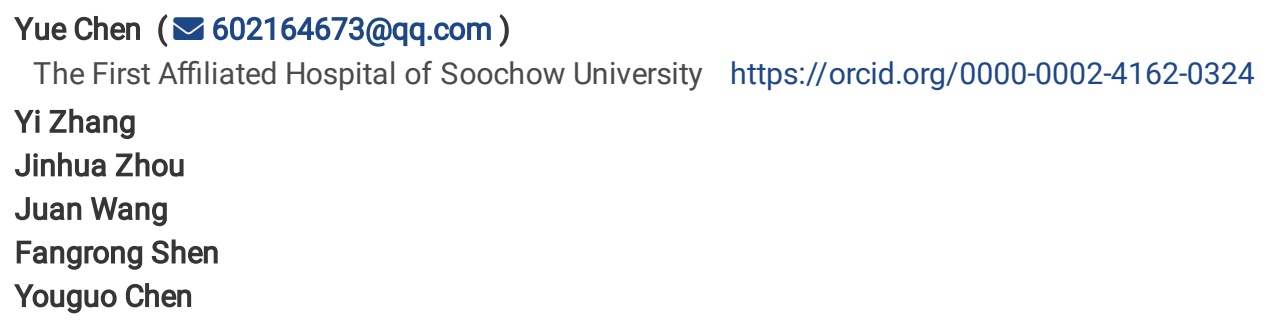

Yue Chen ( $D$ 602164673@qq.com )

The First Affiliated Hospital of Soochow University https://orcid.org/0000-0002-4162-0324

Yi Zhang

Jinhua Zhou

Juan Wang

Fangrong Shen

Youguo Chen

Research Article

Keywords: WT1, P53, Ki-67, ovarian cancer, recurrence

Posted Date: November 4th, 2021

DOI: https://doi.org/10.21203/rs.3.rs-1048180/v1

License: (c) (i) This work is licensed under a Creative Commons Attribution 4.0 International License. Read Full License 


\section{Abstract}

Purpose: To evaluate the ability of WT1 alone, and in combination with P53/Ki-67, to predict recurrence in patients with epithelial ovarian cancer (EOC).

Methods: In this retrospective study, we performed immunohistochemistry on surgical specimens acquired from 137 patients with EOC. We analyzed the sensitivity of WT1 alone, and in combination with P53 and Ki-67, to predict the recurrence of EOC by applying Chi-squared and layered Chi-squared tests. The Kaplan-Meier method was used to predict progression-free survival(PFS).

Results: The proportion of WT1-positive patients in the recurrent group (80.4\%) was significantly higher than that in the non-recurrent group (60.4\%; $\mathrm{P}=0.019)$. In stratified cohort analysis, the recurrence rate of WT1-positive patients was significantly higher than that of WT1-negative patients in the P53-positive group ( $P=0.034)$; similar results were obtained for the group with Ki-67 index $>30 \%(P=0.026)$. Kaplan-Meier curves showed that WT1-negative patients presented with a significantly longer PFS than WT1-positive patients in the P53(P=0.006) and Ki67( $P=0.016)$ positive groups.

Conclusion: A high level of WT1 is a potent predictor of recurrence for EOC. Our analyses showed that the predictive ability of WT1 was more sensitive when combined with P53/Ki-67. Monitoring the levels of WT1 could have significant therapeutic implications for patients with EOC.

\section{Introduction}

Epithelial ovarian cancer (EOC) is a gynecological tumor with the highest degree of malignancy and a poor prognosis. The 5-year survival rate of patients with EOC is only $30 \%$ [1]. An important factor affecting the prognosis of EOC is the high recurrence rate; previous research has shown that most patients with EOC will eventually relapse within two years of treatment [2]. The main factors responsible for the recurrence of EOC is the high degree of malignancy, poor differentiation, and rapid proliferation [3]. P53 and Ki-67 are common clinical immunopathological indicators of tumor cell proliferation and differentiation [4, 5]. However, these two indicators do not have strong

sensitivity [6]. In addition to P53 and Ki-67, Wilms Tumor 1 (WT1) has been used as an immunological marker for the pathological detection of a variety of tumors over recent years. The WT1 gene has been identified as a tumor suppressor gene in Wilms tumors; however, there is increasing evidence that WT1 is overexpressed and exerts an oncogenic role in different types of cancers, including leukemia and myelodysplastic syndrome [7], lung cancer [8], and breast cancer [9]. Although WT1 is a known pathological marker for EOC, it is not clear whether the expression levels of WT1 are specifically related to recurrence.

In this study, we used stratified cohort analysis to investigate whether WT1 expression alone, or in combination with P53 and Ki-67, could predict recurrence in patients with EOC.

\section{Materials And Methods}

Patients and materials: This study included a total of 137 patients undergoing surgery for EOC in the First Affiliated Hospital of Soochow University from 1st January 2015 with follow-up dates to 31st March 2020. The study was approved by the Institutional Ethics Review Board of the Soochow University School of Medicine. Because this was a retrospective study, and the names of patients were not divulged, the Institutional Ethics Review Board waived the need for informed consent. All surgeries were performed by accredited gynecological oncologists; these physicians also determined the residual tumor size. Pathological analysis revealed that all patients had EOC; 102 patients received 6 or more cycles of chemotherapy. Thirty-five patients did not complete chemotherapy due to other basic diseases or serious complications. Chemotherapy was mainly performed with Paclitaxel + Platinum (Cisplatin or Carboplatin). The period of chemotherapy was approximately 21 days. Five patients received targeted therapy (Olaparib and Niraparib). Neoadjuvant chemotherapy (NACT) was performed with Paclitaxel + Platinum drugs. Disease stage was assigned in accordance with the International Federation of Gynecology and Obstetrics (FIGO) surgical staging criteria. Histological grades were categorized as either high or low. The clinicopathological features are summarized in Table 1.

Immunohistochemistry: All specimens were fixed in formalin, embedded in paraffin, serially sectioned at a thickness of $5 \mu \mathrm{m}$, and mounted on electrostatically charged slides. Paraffin sections were then dewaxed and dehydrated before being incubated with $3 \% \mathrm{H} 2 \mathrm{O} 2 \mathrm{at}$ room temperature for $15 \mathrm{~min}$ to remove endogenous peroxidase. Citrate buffer solution ( $0.1 \mathrm{~mol} / \mathrm{L}, \mathrm{pH} 6.0)$ was then applied for 10 min in a microwave for antigen retrieval. Sections were then blocked with normal goat serum for $15 \mathrm{~min}$ at room temperature. Then, sections were incubated with rabbit antihuman WT1 monoclonal antibody (Ab89901, Abcam, 1:100 dilution), rabbit anti-human P53 monoclonal antibody (Ab32389, Abcam,1:100 dilution), or rabbit anti-human Ki-67 monoclonal antibody (Ab92742, Abcam, 1:100 dilution) at $4^{\circ} \mathrm{C}$ overnight and rinsed three time with PBS (5 min per wash). The following morning, sections were washed and incubated with a biotinylated goat anti-rat IgG secondary antibody (1:200 dilution) at $37^{\circ} \mathrm{C}$ for $1 \mathrm{~h}$. Sections were then rinsed three times in PBS (5 min per wash) and positive antibody binding was visualized by DAB color development. Finally, sections were stained in hematoxylin, hydrated with a gradient series of alcohols, cleared with xylene, sealed onto glass slides with neutral gum, and observed by microscopy.

Page 2/9 
Statistical analyses: All statistical analyses were performed with SPSS version 25.0 (IBM Corp., Armonk, NY, USA). The association of WT1 with specific clinical characteristics was analyzed by the t test and by Chi-squared tests. The association between WT1, P53, and Ki67 expression, and the recurrence of EOC was analyzed by layered Chi-squared tests. Kapla-Meier survival curves were used to determine progression-free survival (PFS). The log-rank test was used to compare survival between different groups. All statistical tests were two-sided and significance was set at $P$ $<0.05$.

\section{Results}

The proportions of patients who were positive and negative for WT1, P53, and Ki-67, were determined by calculating the proportions (\%) of the total number of tumor cells that showed positive antibody staining in the nuclei (Figure 1). Statistical analyses showed that WT1-positive staining was present in 92/137 of specimens, P53-positive staining was observed in 92/137 of specimens, and positive Ki-67 staining (>30\%) was observed in 106/137 of specimens. The characteristics of the patients in each group are shown in Table 1.

The mean age of the study population in the WT1-positive group was 53.54 9 9.58years (range: 27.3-75.5 years). In the WT1-positive group ( $\mathrm{N}=92), 58 / 92$ (63.04\%) were postmenopausal, 38/92 (41.30\%) had more than $500 \mathrm{ml}$ of ascites, $60 / 92$ (65.2\%) had no residual tumor, and $20 / 92$ $(21.7 \%)$ were treated by NACT. In total, $92.4 \%$ of patients presented with grade 3 tumors and $83.7 \%$ presented with stage $\nabla-\otimes$ tumors. Analysis also showed that $70.7 \%$ of patients were P53-positive and $80.4 \%$ were positive for Ki-67>30\%. In the WT1-negative group (N=45), the mean age at diagnosis was $52.91 \pm 11.37$ years (range: $24.2-74.8$ years); $25 / 45$ (55.56\%) were postmenopausal, $9 / 45$ (20.0\%) had more than $500 \mathrm{ml}$ of ascites, $36 / 45$ (86.7\%) had no residual tumor, and $4 / 45$ (8.9\%) were treated by NACT. In total, $60 \%$ of patients presented with grade 3 tumors and $53.5 \%$ had stage $\nabla-\nabla$ tumors. Analysis showed that $62.2 \%$ of patients were P53-positive and 71 . $1 \%$ were positive for Ki-67>30\%.

Next, the expression of biomarkers in the recurrent and non-recurrent patients were compared by Chi-squared analysis (Table 2). The proportion of WT1-positive patients in the recurrent group (80.4\%) was significantly higher than that in the non-recurrent group (60.4\%; $\mathrm{P}=0.019)$. There was no significant difference in P53-positive expression when compared between the recurrent (63.0\%) and non-recurrent group (70.3\%; $\mathrm{P}=0.388)$. Similarly, the there was no significant difference in Ki-67-positive (>30\%) expression when compared between the recurrent (80.4\%) and nonrecurrent group $(75.8 \%)(P=0.542)$.

Next, we performed stratified Chi-squared analysis for recurrence (Table 3). In P53-positive patients, the recurrence rate of WT1-positive patients (40\%) was significantly higher than for WT1-negative patients (10.7\%; P=0.034). Similarly, the recurrence rate of WT1-positive patients (43.2\%) was significantly higher than that for WT1-negative patients (15.6\%) in Ki-67-positive (>30\%) patients $(P=0.026)$. However, there was no significant difference between P53-negative and Ki-67-positive ( $\leq 30 \%)$ patients.

Next, the correlation between P53/Ki-67 positivity, WT1-positivity, and progression-free survival (PFS), was evaluated in patients with EOC by Kaplan-Meier curves (Figure 2). Survival analysis included 93 P53-positive patients; 65 patients were WT1-positive, and 28 patients were WT1negative. The PFS of WT1-positive patients was 25.49 months; this compared to 31.57 months in WT1-negative patients. Kaplan-Meier curves showed that P53-positive and WT1-negative patients presented with a significantly longer PFS than WT1-positive patients [Log-rank P $=0.006$ ]. Similarly, survival analysis included 106 Ki-67-positive (>30\%) patients: 74 patients were WT1-positive, and 32 patients were WT1-negative. The PFS of WT1-positive patients was 25.37 months; this compared to 29.98 months in WT1-negative patients. Kaplan-Meier curves showed that Ki67-positive (>30\%) and WT1-negative patients presented with a significantly longer PFS than WT1-positive patients [Log-rank P = 0.016].

\section{Discussion}

Because of its high recurrence rate and poor prognosis, EOC is considered to be the gynecological tumor with the highest degree of malignancy. At present, the clinical judgment of EOC recurrence depends mainly on an increase in tumor indicators and imaging changes. However, there are no valid methods to predict the recurrence of EOC. Since there is no definitively effective treatment for recurrent patients, these patients have a very poor prognosis.

The WT1 gene, located on chromosome 11p13, was originally identified as a tumor suppressor gene in Wilms tumors in children [10]. Since then, WT1 has been identified as an oncogenic factor in a variety of human cancers, including leukemia and myelodysplastic syndrome [11], lung cancer [8], and breast cancer [12]. It has been reported that WT1 enhances the migration and invasive capacity of EOC cells and could be associated with a poor prognosis. However, it is not clear whether WT1 expression is related to recurrence. In the current study, the proportion of WT1-positive patients in the recurrent group was significantly higher than that in the non-recurrent group. Many previous studies have reported that epithelial ovarian tumors generally show high levels of WT1 gene expression [13, 14]. Han et al. reported that WT1 enhanced the migration and invasion capacity of EOC cells [15]. Furthermore, Taube et al. reported that patients with high expression levels of WT1 were more likely to relapse [16]. There is increasing evidence that WT1 exerts an oncogenic role, rather than a tumor-suppressive role,in the development and progression of cancers. Therefore, the positive expression of WT1 may cause higher recurrence in EOC patients. 
It is notable that we detected patients without recurrence in the WT1-positive group. To explore which subgroups were more sensitive to WT1 with regards to predicting recurrence, we performed stratified cohort analysis. Our data showed that patients with combined WT1-positivity and P53/Ki-67-positivitity (>30\%) were more likely to relapse. In a previous study, Bani-Ahmad et al. reported that the enhanced expression levels of WT1 and P53 play a negative role in the pathogenesis and progression of acute promyelocytic leukemia [17]. Similar results were reported for ovarian cancer by Carter et al. who investigated partial or total P53 tumor functional inactivation; under these conditions, WT1 could be overexpressed to promote tumor growth [18]. In another study, Mondal et al. reported that the expression levels of Ki-67 and WT1 may represent indicators of an unfavorable prognosis in patients with advanced serous epithelial ovarian carcinoma [19]. However, these authors did not investigate whether the combination of these factors could indicate a poor prognosis. Further studies on the association between Ki- 67 and WT1 expression are now required.

Our analysis showed that WT1-negative patients presented with a significantly longer PFS than WT1-positive patients in the P53-positive and Ki67-positive (>30\%) groups. Our data also indicated that WT1 gene expression, in combination with p53 and Ki-67 expression, is significantly associated with PFS. Many previous studies have suggested that WT1 expression alone is associated with PFS [20, 21]. Qi et al. carried out metaanalysis involving 3620 cases and found that WT1-positive expression was significantly associated with a worse overall survival (OS) and PFS [22]. However, previous studies have failed to consider whether the combination of WT1, P53 and Ki-67 expression is related to PFS; further studies are now required to address this possibility.

\section{Conclusion}

The results of the present study suggest that WT1 is associated with the recurrence of EOC and that WT1-positive patients are more likely to relapse. The combination of WT1 expression with P53 or Ki-67 expression represents an efficient prognostic indicator for EOC patients. WT1positive patients presented with a significantly shorter PFS than WT1-negative patients in the P53-positive and Ki-67-positive (>30\%) groups. In future, the targeting of WT1 could have therapeutic implications for the treatment of EOC.

\section{Declarations}

\section{Author Contributions}

YC and FS contributed to the study design, literature search, and data acquisition and interpretation. YC and $Y Z$ performed the major data analyses, statistical analysis, and manuscript editing. JW and JZ contributed to data acquisition, statistical analysis, and manuscript revision.

\section{Funding}

This work was supported by National Natural Science Foundation of China(No.81772773)

\section{Acknowledgments}

We thank the clinicians of the Department of Obstetrics and Gynecology at the First Affiliated Hospital of Soochow University for their assistance.

\section{Statements and Declarations}

\section{Conflicts of Interest}

The authors declare that there are no conflicts of interest with regards to the publication of this article.

Ethical approval and consent to practice The specimens were collected for histopathological diagnostics and used for the study after no clinical use was indicated any longer.Approval was obtained from the Institutional Ethics Review Board of the Soochow University School of Medicine. The procedures used in this study adhere to the tenets of the Declaration of Helsink

\section{References}

1. Herrera FG, Irving M, Kandalaft LE, Coukos G: Rational combinations of immunotherapy with radiotherapy in ovarian cancer.The Lancet Oncology. 2019 Aug;20(8):e417-e433.https://doi.org/10.1016/S1470-2045(19)30401-2 .

2. Klotz DM, Wimberger P. : Overcoming PARP inhibitor resistance in ovarian cancer: what are the most promising strategies?Archives of Gynecology Obstetrics.2020,302(5):1087-1102. https://doi.org/10.1007/s00404-020-05677-1.

3. tewart CS, Ralyea C, Lockwood S: Ovarian Cancer: An Integrated Review. Seminars in Oncology Nursing.2019,Apr;35(2):151-156. https://doi.org/10.1016/j.soncn.2019.02.001. 
4. Guan YS, He Q, Zou Q: Status quo of p53 in the treatment of tumors. Anti-cancer drugs.2016, 27(9):811.

https://doi.org/10.1097/CAD.0000000000000397.

5. Sun, Xiaoming, Kaufman, Paul: Ki-67: more than a proliferation marker. Chromosoma.2018,06 ;127(2): $175-186$.

https://doi.org/10.1007/s00412-018-0659-8.

6. Giurgea LN, Ungureanu C, Mihailovici MS: The immunohistochemical expression of p53 and Ki67 in ovarian epithelial borderline tumors.Correlation with clinicopathological factors. Romanian journal of Morphology and Embryology.2012, 53(4):967.

7. Mitrovic M, Kostic T, Virijevic M, Karan-Djurasevic T, Vukovic NS ect al: The influence of Wilms' tumor 1 gene expression level on prognosis and risk stratification of acute promyelocytic leukemia patients. International Journal of Laboratory Hematology.2020 Feb;42(1):82-87. https://doi.org/10.1111/ijlh.13144.

8. Seiji, Hayashi, Yusuke, Oji, Yuko, Kanai, Tomoaki ect al: Low Wilms' Tumor Gene Expression in Tumor Tissues Predicts Poor Prognosis in Patients with Non-Small-Cell Lung Cancer.Cancer Investigation. Vol 30, No 2. 2011. https://doi.org/10.3109/07357907.2011.633291.

9. Jiang, Jun.High Wilms' tumor 1 mRNA expression correlates with basal-like and ERBB2 molecular subtypes and poor prognosis of breast cancer.Oncology Reports.2012,Oct;28(4):1231-6. https://doi.org/10.3892/or.2012.1906.

10. Hastie N.D.Wilms' tumour 1 (WT1) in development, homeostasis and disease. Development.2017, 144(16):2862-2872. https://doi.org/10.1242/dev.153163..

11. Israyelyan A, Goldstein L, Tsai W, Aquino L, Forman SJ, Nakamura R.Real-time assessment of relapse risk based on the WT1 marker in acute leukemia and myelodysplastic syndrome patients after hematopoietic cell transplantation.Bone Marrow Transplantation.2015 Jan;50(1):26-33. https://doi.org/10.1038/bmt.

12. Zhang Y, Yan WT, Yang ZY, Li YL, Qi X.W.The role of WT1 in breast cancer: clinical implications, biological effects and molecular mechanism. International Journal of Biological Sciences.2020,16(8):1474-1480. https://doi.org/10.7150/ijbs.39958.

13. Barbolina MV, Adley BP, Shea LD, Stack M.S.Wilms tumor gene protein 1 is associated with ovarian cancer metastasis and modulates cell invasion. Cancer.2008, 112(7):1632-1641.https://doi.org/10.1002/cncr.23341.

14. Yu HL, Ma XD, Tong JF, Li JQ, Guan XJ.WTAP is a prognostic marker of high-grade serous ovarian cancer and regulates the progression of ovarian cancer cells. Onco Targets and Therapy.2019 Aug 6;12:6191-6201. https://doi.org/10.2147/OTT.S205730.

15. Han, Song, Zhang, Zhou, Zhang Wang, ect al.Wilms' tumor 1 (WT1) promotes ovarian cancer progression by regulating E-cadherin and ERK1/2 signaling. Cell Cycle.2020,Oct;19(20):2662-2675. https://doi.org/10.1080/15384101.2020.1817666.

16. Denkert, Carsten, Letsch,ect al.Wilms tumor protein 1 (WT1) - Not only a diagnostic but also a prognostic marker in high-grade serous ovarian carcinoma.Gynecologic Oncology.2016.Mar ;140(3): 494-502. https://doi.org/10.1016/j.ygyno.2015.12.018.

17. Bani-Ahmad MA, Al-Sweedan SA, Al-Asseiri MA, Alkhatib AJ. :A Proposed Kinetic Model for the Diagnostic and Prognostic Value of WT1 and p53 in Acute Myeloid Leukemia. Clinical laboratory.2018, 64(3):357-363. https://doi.org/10.7754/Clin.Lab.2017.170915.

18. Julia H Carter,James A Deddens,Gretchen Mueller,ect al.Transcription factors WT1 and p53 combined: a prognostic biomarker in ovarian cancer.British Journal of Cancer.2018,Aug;119(4):462-470. https://doi.org/10.1038/s41416-018-0191-x.

19. Mondal SK, Basak B, Bhattacharya S, Panda.Role of WT1, B-cell lymphoma 2, Ki-67 (Mib1), and Her2/Neu as diagnostic and prognostic immunomarkers in ovarian serous and endometroid carcinoma.Journal of Cancer Research and Therapeutics.2021, 17(1):166.

https://doi.org/10.4103/jcrt.JCRT_311_19.

20. Andersson C, Oji Y, Ohlson N, Wang S. Prognostic significance of specific anti-WT1 IgG antibody level in plasma in patients with ovarian carcinoma. Cancer Medicine.2014, 3(4).https://doi.org/10.1002/cam4.244.

21. Liu Z, Yamanouchi K, Ohtao T, Matsumura S, Kurachi H.High Levels of Wilms' Tumor 1 (WT1) Expression Were Associated with Aggressive Clinical Features in Ovarian Cancer. Anticancer Research.2014, 34(5):2331-2340.

22. Qi XW, Zhang F, Wu H, Liu JL, Zong BG, Xu C, Jiang JJR: Wilms' tumor 1(WT1) expression and prognosis in solid cancer patients: a systematic review and meta-analysis. Scientific Reports. 2015 Mar 9;5:8924. https://doi.org/10.1038/srep08924.

\section{Tables}


Table 1 Association of WT1 with the clinical characteristics of epithelial ovarian cancer

Patient characteristics

\begin{tabular}{|c|c|c|c|}
\hline Characteristics $\rrbracket$ & WT1 positive & WT1 negative & P-Values \\
\hline & $\mathrm{N}=92$ & $\mathrm{~N}=45$ & \\
\hline \multicolumn{4}{|l|}{ Age $\mathbb{Z}$} \\
\hline Avarege & $53.54(27.3-75.5)$ & $52.91(24.2-74.8)$ & 0.734 \\
\hline Menopause & & & 0.400 \\
\hline No & $34(36.96 \%)$ & $20(44.44 \%)$ & \\
\hline Yes & $58(63.04 \%)$ & $25(55.56 \%)$ & \\
\hline Volume of ascites $₫ \mathrm{ml} \rrbracket$ & & & 0.014 \\
\hline$\leq 500$ & $54(58.70 \%)$ & $36(80 \%)$ & \\
\hline$>500$ & $38(41.30 \%)$ & $9(20 \%)$ & \\
\hline \multicolumn{4}{|l|}{$\mathrm{RTa}$} \\
\hline ROb & $60(65.2 \%)$ & $39(86.7 \%)$ & 0.008 \\
\hline R1c & $32(34.8 \%)$ & $6(13.3 \%)$ & \\
\hline \multicolumn{4}{|l|}{ NACTd } \\
\hline Yes & $20(21.7 \%)$ & $4(8.9 \%)$ & 0.063 \\
\hline No & $72(78.2 \%)$ & $41(91.1 \%)$ & \\
\hline \multicolumn{4}{|l|}{ FIGOe stage } \\
\hline$\bigotimes \sim \bigotimes$ & $15(16.3 \%)$ & $21(46.7 \%)$ & 00.001 \\
\hline$\nabla \sim \bigotimes$ & $77(83.7 \%)$ & $24(53.3 \%)$ & \\
\hline \multicolumn{4}{|l|}{ Histologic grade } \\
\hline Grade 1-2f & $7(7.6 \%)$ & $18(40 \%)$ & $\square 0.001$ \\
\hline Grade $3 g$ & $85(92.4 \%)$ & $27(60 \%)$ & \\
\hline \multicolumn{4}{|l|}{ P53 } \\
\hline Positive & $65(70.7 \%)$ & $28(62.2 \%)$ & 0.321 \\
\hline Negative & $27(29.3 \%)$ & $17(37.8 \%)$ & \\
\hline \multicolumn{4}{|l|}{ Ki-67 } \\
\hline$>30 \%$ positive & $74(80.4 \%)$ & $32(71.1 \%)$ & 0.221 \\
\hline$\leq 30 \%$ positive & $18(19.6 \%)$ & $13(28.9 \%)$ & \\
\hline
\end{tabular}

Values are expressed as avarege (minimum-maximum) or number (percentage).

aRTヌpresence of residual tumor

$\mathrm{bR0}=$ no RT or RT smaller than $10 \mathrm{~mm}{ }^{\mathrm{c}} \mathrm{R} 1=\mathrm{RT}$ higher than $10 \mathrm{~mm}$;

dNACT:Neoadjuvant chemotherapy

eFIGO: The International Federation of Gynecology and Obstetrics(2021): I:Tumor limited to ovaries (one or both) or fallopian tube(s);

II:Tumor involves one or both ovaries or fallopian tubes with pelvic extension below pelvic brim or primary peritoneal cancer;

III:Tumor involves one or both ovaries or fallopian tubes, or primary peritoneal cancer, with microscopically confirmed peritoneal metastasis outside the pelvis and/or metastasis to the retroperitoneal (pelvic and/or para-aortic) lymph nodes; 
IV:Distant metastasis, including pleural effusion with positive cytology; liver or splenic parenchymal metastasis; metastasis to extra-abdominal organs (including inguinal lymph nodes and lymph nodes outside the abdominal cavity); and transmural involvement of intestine.

fGrade 1-2:Grade1:High differentiated;Grade2:Moderately differentiated

gGrade 3:Low differentiated

Table 2 Markers for the recurrence of epithelial ovarian cancer

\begin{tabular}{|llll|}
\hline & Recurrence & Non-recurrence & P-values \\
\cline { 2 - 4 } WT1 & $\mathrm{N}=46$ & $\mathrm{~N}=91$ & \\
\hline Positive & $37(80.4 \%)$ & $55(60.4 \%)$ & $\mathbf{0 . 0 1 9}$ \\
\hline Negative & $9(19.6 \%)$ & $36(39.6 \%)$ & $\mathbf{0 . 0 1 9}$ \\
\hline P53 & & & \\
\hline Positive & $29(63.0 \%)$ & $64(70.3 \%)$ & 0.388 \\
\hline Negative & $17(27.0 \%)$ & $27(29.7 \%)$ & 0.388 \\
\hline Ki-67 & & & \\
\hline$>30 \%$ positive & $37(80.4 \%)$ & $69(75.8 \%)$ & 0.542 \\
\hline$\leq 30 \%$ positive & $9(19.6 \%)$ & $22(24.2 \%)$ & 0.542 \\
\hline
\end{tabular}

Values are expressed as number (percentage).

Table 3 Stratified analysis of the relationship between WT1 and recurrence

\begin{tabular}{|lllll|}
\hline & WT1 positive & WT1 negative & P-Values & RR(95\%Cl) \\
& $\mathrm{N}=92$ & $\mathrm{~N}=45$ & & \\
\hline P53 & & & & \\
\hline Positive & $26 / 65(40 \%)$ & $3 / 28(10.7 \%)$ & $\mathbf{0 . 0 3 4}$ & $3.733(1.230-11.327)$ \\
\hline Negative & $11 / 27(40.7 \%)$ & $6 / 17(35.3 \%)$ & 0.761 & $1.154(0.525-2.539)$ \\
\hline Ki-67 & & & & \\
\hline$>30 \%$ positive & $32 / 74(43.2 \%)$ & $5 / 32(15.6 \%)$ & 0.026 & $2.768(1.187-6.452)$ \\
\hline$\leq 30 \%$ positive & $5 / 18(27.8 \%)$ & $4 / 13(30.8 \%)$ & 1.000 & $0.903(0.299-2.724)$ \\
\hline
\end{tabular}

Values are expressed as number (percentage).

\section{Figures}




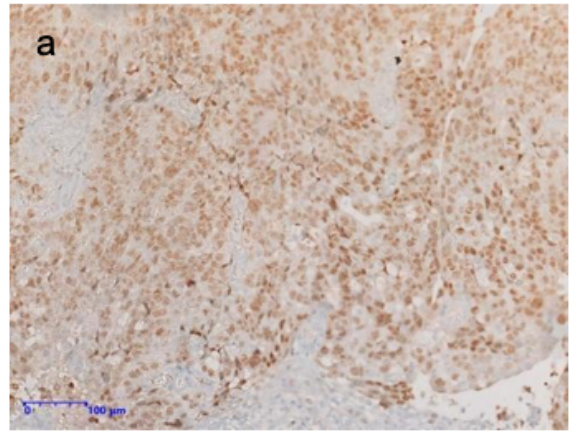

WT1 positive

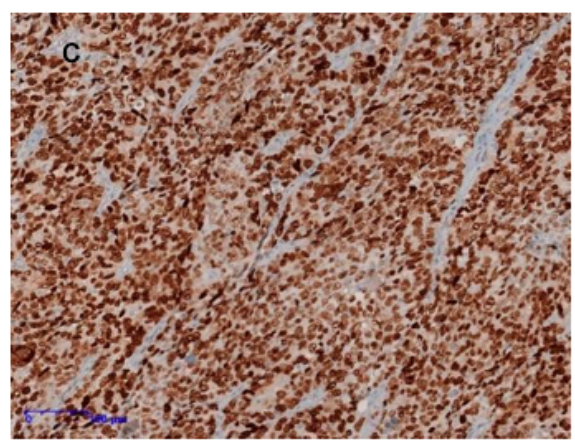

P53 positive

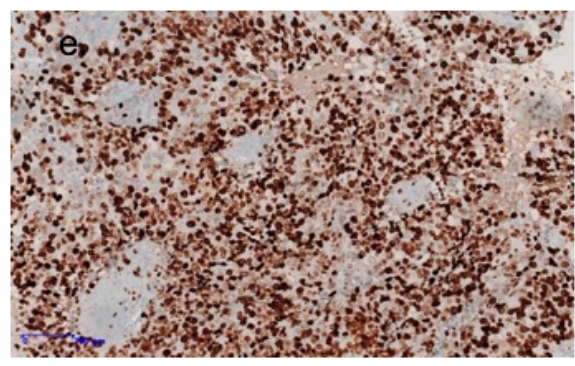

Ki-67>30\%positive

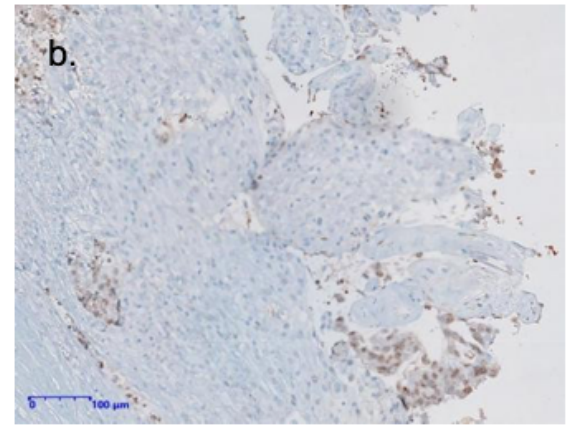

WT1 negative

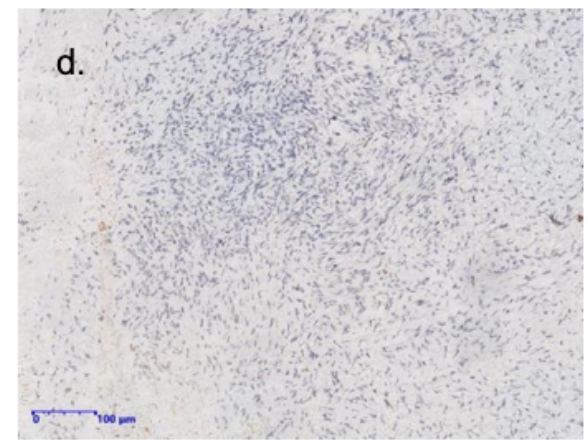

\section{P53 negative}

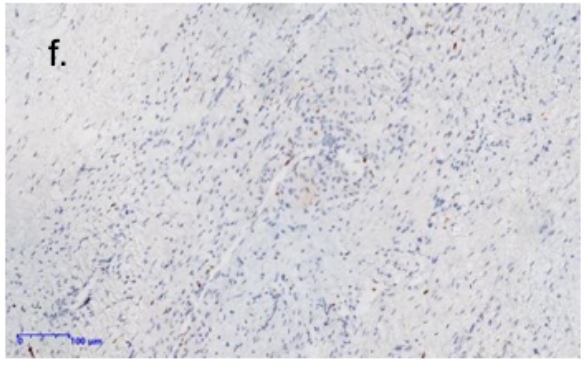

Ki-67 $\leq 30 \%$ positive

\section{Figure 1}

Immunohistochemistry results of WT1هP53 and Ki-67 expression in ovarian tissues. Representative images of WT1, P53 and Ki-67 immunohistochemical expression in epithelial ovarian cancer. (a) positive expression of WT1 (5x); (b) negative expression of WT1 (5x); (c) positive expression of P53 (5x); (d) negative expression of P53 (5x); (e) >30\% positive expression of Ki-67 (5x); (g) $\leq 30 \%$ positive expression of Ki-67 (5x).

a.

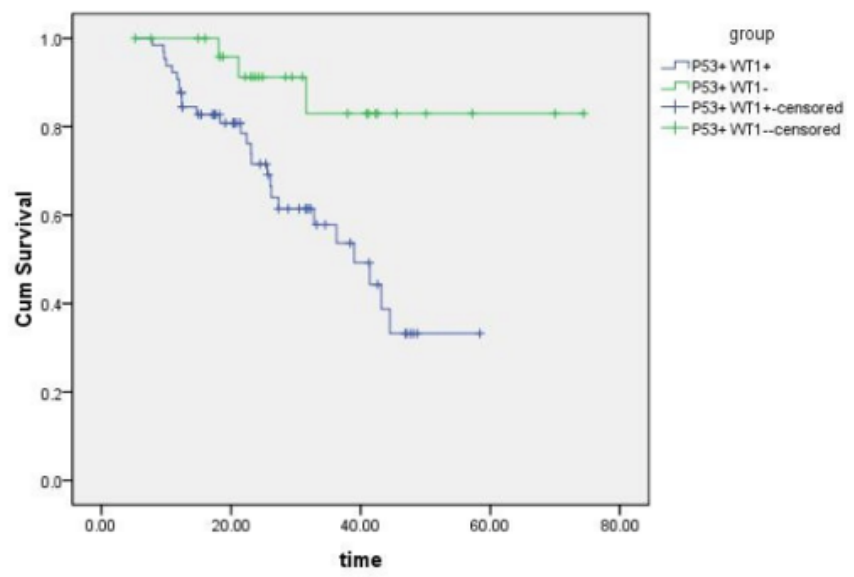

b. Survival Functions

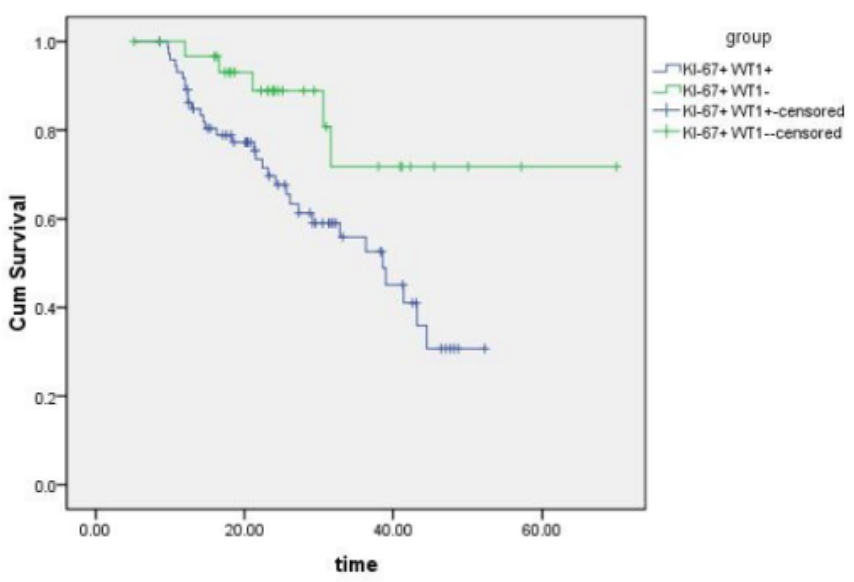


Kaplan-Meier curves for progression-free survival(PFS). Kaplan-Meier curves of progression-free survival (PFS) probability in P53 and Ki-67 positive ovarian cancer patients with WT1 positivity(blue) as compared to those who were WT1 negative (green). 